\title{
Legendre spectral finite elements for Reissner-Mindlin composite plates
}

\author{
Michael A. Sprague ${ }^{\mathrm{a}, *}$, Avi Purkayastha ${ }^{\mathrm{a}}$ \\ ${ }^{a}$ Computational Science Center, National Renewable Energy Laboratory, Golden, CO \\ 80401, USA
}

\begin{abstract}
Legendre spectral finite elements (LSFEs) are examined in their application to Reissner-Mindlin composite plates for static and dynamic deformation on unstructured grids. LSFEs are high-order Lagrangian-interpolant finite elements whose nodes are located at the Gauss-Lobatto-Legendre quadrature points. Nodal quadrature is employed for mass-matrix calculations, which yields diagonal mass matrices. Full quadrature or mixed-reduced quadrature is used for stiffness-matrix calculations. Solution accuracy is examined in terms of model size, computation time, and memory storage for LSFEs and for quadratic serendipity elements calculated in a commercial finite-element code. Linear systems for both model types were solved with the same sparsesystem direct solver. At their best, LSFEs provide many orders of magnitude more accuracy than the quadratic elements for a fixed measure (e.g., computation time). At their worst, LSFEs provide the same accuracy as the quadratic elements for a given measure. The LSFEs were insensitive to shear locking and were shown to be more robust in the thin-plate limit than their low-order counterparts.
\end{abstract}

Keywords: composite; finite element; high order; numerical methods; Reissner-Mindlin

\section{Introduction}

In this paper we examine the performance of Legendre spectral finite elements (LSFEs) for calculating the static and dynamic response of composite

\footnotetext{
${ }^{*}$ Corresponding Author

Email address: Michael.A.Sprague@nrel.gov (Michael A. Sprague)

Preprint submitted to Finite Elements in Analysis and Design

June 19, 2015
} 
Reissner-Mindlin (RM) plates. RM plate and shell models are commonly used in commercial finite element (FE) codes. Examples include the SHELL181 and SHELL281 elements in ANSYS ${ }^{\circledR}$ [1] and the S4R element in Abaqus ${ }^{\circledR}$ [2]. LSFEs are $p^{\text {th }}$-order Lagrangian-interpolant FEs whose nodes are located at the $p+1$ Gauss-Lobatto-Legendre (GLL) points. LSFEs can provide exponential (i.e., spectral) convergence rates through $p$-refinement while maintaining the geometric-modeling capabilities of low-order FE methods. The motivation for using LSFEs over low-order FEs can be stated from two perspectives. For a given computational effort (either in terms of model size or computing time), LSFEs can provide dramatically more accurate solutions than low-order elements. Alternatively, if one requires a certain accuracy level, LSFEs can require dramatically less computational effort.

Spectral finite elements were first introduced by Patera [3] for computational fluid dynamics, where the nodes of the Lagrangian-interpolant basis functions were located at the Gauss-Lobatto-Chebyshev (GLC) points. Ronquist and Patera [4] introduced LSFEs with nodes located at the GLL points for fluid dynamics. Since their inception, LSFEs have seen continued development and application in many disciplines (e.g., fluids, acoustics, geophysics). However, their application to structural dynamics has been comparatively limited (see, e.g., Brito and Sprague [5] for more discussion).

LSFEs were first applied to isotropic RM plates by Zrahia and Bar-Yoseph [6], where all FE inner products were evaluated with GLL nodal quadrature. While nodal quadrature is a reduced quadrature scheme for RM-plate mass and stiffness matrices, it is appealing because it is sufficiently accurate to maintain the order of convergence of the method [7], it provides consistent diagonal mass matrices [8], and GLL quadrature requires less computational effort than Gauss-Legendre quadrature for evaluation of stiffness-matrix inner products. Zrahia and Bar-Yoseph [6] showed, on a rectangle-domain mesh composed of a single square element, exponential convergence rates for static-deformation and natural-frequency problems. They also found, for their test case, that the LSFEs were shear-locking free. Kudela et al. [9] applied LSFEs in space and time to wave propagation in RM composite plates, where square elements were used along with nodal quadrature. Moleiro et al. [10, 11] applied LSFEs in a least-squares formulation for RM plates where static deformation and natural frequencies were calculated on a structured grid for a rectangular plate. Unlike Zrahia and Bar-Yoseph [6], who employed nodal quadrature, Moleiro et al. [10, 11] employed full Gauss-Legendre quadrature for mass and stiffness inner products. Again, 
solutions were found to be insensitive to shear locking. Liu et al. [12] examined Mindlin spectral elements for isotropic plates where the nodes were located at the Chebyshev-Gauss-Lobatto points, and both nodal and GuassLegendre quadrature were examined for stiffness matrix calculations but only nodal quadrature was used for mass matrices, which were in turn diagonal. As with Zrahia and Bar-Yoseph, shear-locking studies were limited to square elements, and both both quadrature schemes produces solutions insensitive to shear locking. The elements described by Liu et al. [12] were used by $\mathrm{Hu}$ et al. [13] to characterize damage size in metallic plates.

Brito and Sprague [5] examined LSFEs applied to the static and dynamic response of isotropic RM plates on an unstructured grid for a circular plate. They demonstrated that LSFEs on an unstructured grid, and whose inner products were calculated with nodal quadrature, were prone to shear locking in the limit of vanishing plate thickness. This was attributed to the non-constant Jacobian of the element mapping. Brito and Sprague [5] introduced a mixed reduced quadrature scheme for which the shear-stiffness inner products were evaluated with reduced Gauss-Legendre quadrature, and bending-stiffness inner products were evaluated with nodal quadrature. This mixed scheme was shown to be locking free and maintained the desired exponential convergence rates. Also examined was the computational cost of LSFEs compared to standard first- and second-order elements for static- and dynamic-deformation problems. LSFEs offered dramatic savings over loworder elements. For the static problems, linear systems were solved with a Jacobi-preconditioned conjugate gradient solver, and for which LSFE matrixvector products were evaluated in a tensor-product-factorized form. For the dynamic problems, central finite differences were employed.

In this paper, we compare LSFEs against the serendipity-type quadratic SHELL281 elements in the commercial code ANSYS for a composite Reissner-Mindlin plate discretized with an unstructured grid. For static deformation, accuracy is examined in terms of model size (number of nodes), required memory storage, and computation wall-clock time for solution of linear systems where the LSFE and SHELL281 systems were solved with the same sparse-system direct solver. For dynamic deformation, the LSFE and SHELL281 models were discretized with a standard implicit trapezoidal rule, and accuracy is compared in terms of model size and computation time. 


\section{Formulation and numerical implementation}

\subsection{Reissner-Mindlin model and Legendre spectral finite elements}

The Reissner-Mindlin plate LSFEs are formulated with a BubnovGalerkin approach. The formulation, where trial and weight functions are the same Lagrangian-interpolant functions, is well established (see, e.g., Hughes [14]). We provide here a brief overview. The fundamental assumptions grounding Reissner-Mindlin plate deformation are that $(i)$ in-plane displacements vary linearly through the plate thickness, $(i i)$ stress in the normal direction is negligible, and (iii) transverse displacement is constant through the thickness.

We are interested in calculating the static and dynamic responses of a plate with constant thickness $h$, whose midplane spans a domain $\Omega$ in the $(x, y)$ plane. For our analyses, external forcing is restricted to a transverse distributed force $f(x, y)$ and boundary conditions are assumed to be clamped unless otherwise noted. The formulation is readily extended to accommodate more general forcing and/or boundary conditions. The generalized displacement vector of the plate midplane is denoted $\mathbf{U}=\left[\begin{array}{lllll}u & v & w & \theta^{y} & \theta^{x}\end{array}\right]^{T}$, where $u(x, y), v(x, y)$, and $w(x, y)$ are the midplane displacements in the $x$, $y$, and $z$ directions, respectively. $\theta^{y}(x, y)$ and $\theta^{x}(x, y)$ are the plate rotations about the $y$ and $x$ axes, respectively.

In our finite-element discretization, $\Omega$ is represented as $n_{e l}$ nonoverlapping quadrilateral elements with straight or curved edges, each with domain $\Omega^{e}$, such that

$$
\Omega \approx \Omega^{h}=\cup_{e=1}^{n_{e l}} \Omega^{e} .
$$

The element domain is given by $\mathbf{x} \in \Omega^{e}$, where

$$
\mathbf{x}=\sum_{\widehat{i}=1}^{(p+1)^{2}} \mathbf{x}_{\hat{i}} \Phi_{\widehat{i}},
$$

where $\mathbf{x}_{\widehat{i}}=\left[\begin{array}{ll}x_{\widehat{i}} & y_{\hat{i}}\end{array}\right]^{T}$ is the location of node $\widehat{i}$, and $\Phi_{\widehat{i}}(x, y)$ is the $\widehat{i}^{\text {th }}$ Lagrangian interpolant based on a tensor product of two one-dimensional $p^{\text {th }}$ order-polynomial Lagrangian interpolants, $\widehat{i} \in\left\{1, \ldots,(p+1)^{2}\right\}$. As described in the Appendix A, the subscript index with a "hat" denotes a lexicographical numbering. In a LSFE formulation, nodes are located at the $(p+1) \times(p+1)$ GLL quadrature points (in element natural coordinates). This node positioning provides optimal polynomial interpolation (with respect to the $L_{2}$ norm) 
[15] and is the fundamental difference between LSFEs and traditional highorder FEs that have nodes evenly spaced throughout the element domain. For the dependent variables, we employ an isoparametric formulation and the generalized-displacement approximation $\mathbf{U}^{h} \approx \mathbf{U}$ is represented (over element domain $\Omega^{e}$ ) as

$$
\mathbf{U}^{h}=\sum_{\widehat{i}=1}^{(p+1)^{2}} \mathbf{U}_{\widehat{i}} \Phi_{\widehat{i}},
$$

where $\mathbf{U}_{\widehat{i}}=\left[\begin{array}{lllll}u_{\hat{i}}^{e} & v_{\hat{i}}^{e} & w_{\hat{i}}^{e} & \theta_{\hat{i}}^{y, e} & \theta_{\widehat{i}}^{x, e}\end{array}\right]^{T}$ is the generalized displacement at node $\widehat{i}$.

The element-level semi-discrete equations governing motion may be written as

$$
\mathbf{M} \ddot{\mathbf{U}}^{h}+\mathbf{K} \mathbf{U}^{h}=\mathbf{F},
$$

where an overdot denotes a time derivative, $\mathbf{M}$ is the mass matrix, $\mathbf{K}$ is the stiffness matrix, and $\mathbf{F}$ is the force vector. For the stiffness-matrix inner products, unless noted otherwise, the mixed-reduced quadrature introduced in Brito and Sprague [5] was employed, where bending- and extensionalstiffness terms were evaluated with $(p+1) \times(p+1)$ nodal quadrature and shear-stiffness terms were evaluated with $p \times p$ Gauss-Legendre quadrature. Mass-matrix inner products were evaluated with nodal quadrature, which resulted in diagonal mass matrices. For comparison studies with low-order elements, stiffness matrices were stored in a compressed-storage-column (CSC) format and only the diagonals of LSFE mass matrices were stored.

One of our numerical studies compares, for static-deformation LSFE systems, the computational cost of sparse-system solution with a preconditioned conjugate-gradient iterative solver. For iterative linear-system solution, the primary computational cost resides in evaluation of matrix-vector products. Matrix-vector products can be efficiently evaluated in two ways. In the first approach the sparse global stiffness matrix is calculated and stored and a sparse matrix-vector-product algorithm can be applied. The LSFE elementstiffness matrix is, in general, full and is $5(p+1)^{2} \times 5(p+1)^{2}$. Hence, the global matrix-vector product requires $O\left(p^{4}\right)$ operations and $O\left(p^{4}\right)$ storage per element. The second approach is "matrix free", where the matrix-vector product is calculated over each element incorporating quadrature and stiffness matrices are never explicitly calculated. In a naive implementation, this requires $O\left(p^{6}\right)$ operations and negligible storage [5]. However, the tensorproduct form can be effectively factorized [16] reducing the matrix-vector 
calculation cost to $O\left(p^{3}\right)$ operations with $O\left(p^{2}\right)$ storage per element [5]. Details regarding the LSFE matrices and their matrix-vector tensor-product factorization are included in Appendix B.

\subsection{Low-order elements}

We are interested in comparing the performance of LSFEs for composite RM plates with that of state-of-the-art low-order elements for static and dynamic linear deformations. To that end we compare against the SHELL281 quadrilateral elements of the commercial FE package ANSYS. SHELL281 elements are serendipity type with eight nodes, and are based on the formulation of Bathe and Dvorkin [17] [1]. The elements have six degrees-of-freedom at each node (including a drilling degree-of-freedom). $2 \times 2$ quadrature is employed in-plane and three quadrature points per layer are employed through the thickness. The material shear stiffness is calculated through an ANSYSproprietary approach. In order to ensure that the SHELL281-element model was solving the same governing RM equations as the LSFE model two modifications were required. First, the drilling degrees of freedom were eliminated and the drilling stiffness was set to zero. Second, the material shear stiffness was calculated directly from the standard RM equations with a shear correction factor of 5/6 (see, e.g., [18], also described in Appendix A). For dynamic simulations, SHELL281 mass matrices were both calculated in consistent and lumped configurations. The global stiffness and mass matrices were exported from ANSYS in Harwell-Boeing (HB) sparse-matrix format [19], which is the CSC format plus additional file-header meta data. Force vectors were also exported from ANSYS.

\subsection{Time discretization}

Dynamic analyses were performed with a standard trapezoidal-rule implicit algorithm (see, e.g., Cook et al. [20]).

\subsection{Numerical implementation, sparse-linear-system solvers, and timing tests}

Linear systems that arose for the static and dynamic FE equations, for both LSFE and ANSYS SHELL281 models, were solved with packages from the HSL Mathematical Software Library [21] coupled with the METIS graphpartitioning libraries. In particular, sparse-system direct solves were accomplished with the HSL_MA97 package, which is based on a multi-frontal method and sparse Cholesky factorization. Indirect sparse-linear-system 
solves were accomplished with the MI28 package, which is a conjugategradient $(\mathrm{CG})$ solver with preconditioning.

LSFE mass and stiffness matrices were calculated with an in-house Fortran 90 code. SHELL281-element mass and stiffness matrices were calculated with ANSYS version 15.0. As described above, LSFE mass matrices were diagonal, whereas SHELL281 mass matrices calculated in either lumped or consistent configurations. For static and dynamic simulation, a Fortran 90 code was created for each that imported mass and stiffness matrices stored in CSC format. This enabled simulations with mass and stiffness matrices created from either the LSFEs or the ANSYS SHELL281 elements. As described above, these codes were equipped with the HSL_MA97 sparse linear-system solver. Having the same code and the same linear-system solvers enabled "apples-to-apples" comparisons between the underlying formulations. A separate Fortran code was used to examine LSFE-model computation time with the HSL MI28 conjugate-gradient solver with default tolerance settings.

For our computational-simulation timing tests, the codes were compiled with the Intel ${ }^{\circledR}$ Fortran compiler version 13.1 .3 with optimization enabled. Simulations were run in serial on a single computational CPU (Intel Xeon CPU, E5-2670) free of other application burdens. For a static simulation, the total computation time was calculated as the elapsed wall-clock time required for matrix factorization (in direct solves) and linear-system solution. For a dynamic simulation, total computation time was calculated as that required for matrix factorization and all subsequent solves at each time step. Total computation time in each configuration was taken as the average of at least five simulations.

\section{Test case: Material, geometry, and FE discretization}

We consider composite plates composed of layers of orthotropic material with properties

$$
\begin{array}{r}
E_{2}=E_{1} / 25 \\
G_{23}=0.2 E_{2}, \quad G_{13}=G_{12}=0.5 E_{2} \\
\nu_{12}=0.25, \quad \nu_{21}=\nu_{12} \frac{E_{2}}{E_{1}}
\end{array}
$$

where $E_{i}$ is the Young's modulus in the $i^{\text {th }}$ direction $(1$ being the fiber direction), $G_{i j}$ is the shear modulus in the $i-j$ plane, and $\nu_{i j}$ is the Poisson's 
ratio for transverse strain in $j$ direction when stressed in the $i$ direction [22]. In our comparison studies, we examine two-layered plates with a $0^{\circ} / 90^{\circ}$ material layup, where the angles are the lamination angles indicating the fiber orientation (in the $(x, y)$ plane) with respect to the positive $x$ axis.

The majority of our numerical studies are for a circular plate with thickness $h$, radius $R$, midplane domain $\Omega$, midplane boundary $\partial \Omega$, and clamped boundary conditions.

$$
u=v=w=\theta^{y}=\theta^{x}=0, \quad \text { on } \quad \partial \Omega .
$$

For verification of our code, we examine below solutions for a square plate with side length $a$ and with boundary conditions described below. We also examine a square plate with clamped-edges and four circular holes of radius $R_{h}$ symmetrically located in the four quadrants of the square domain.

For all simulations full plates were discretized. Figure 1(a) shows the upper right quadrant of our 12-element base LSFE mesh for the circular plate with $p=2$ refinement. LSFE refinement is accomplished by increasing $p$ on the base mesh. Figure 1(b) shows a refined LSFE mesh with $p=6$. As described above, the LSFE nodes are located at the $(p+1) \times(p+1)$ GLL points, which has nodes clustered near element boundaries as shown in Fig. 1(b). The base SHELL281-element mesh was identical to the base LSFE mesh (but without the element-center nodes). Refinement of the SHELL281element model was accomplished by dividing the base-mesh into additional elements. Figure 2 shows the upper right quadrant of our 32-element base LSFE mesh for the perforated square plate with $p=2$ refinement and $a / R_{h}=$ 5. Refinement of the perforated-square-plate mesh was accomplished in the same manner as for the circle-plate mesh for both LSFE and SHELL281element models.

\section{Results}

\subsection{Algorithm/implementation verification}

We consider first verification of our implementation of the LSFEs for composite RM plates as well as our ANSYS SHELL281 implementation. To that end, we examine the static deformation of a simply supported two-layercomposite square plate with side length $a$ and thickness $h=a / 10$ under a uniform distributed load $q_{0}$ as described by Reddy [22]. The materials are 


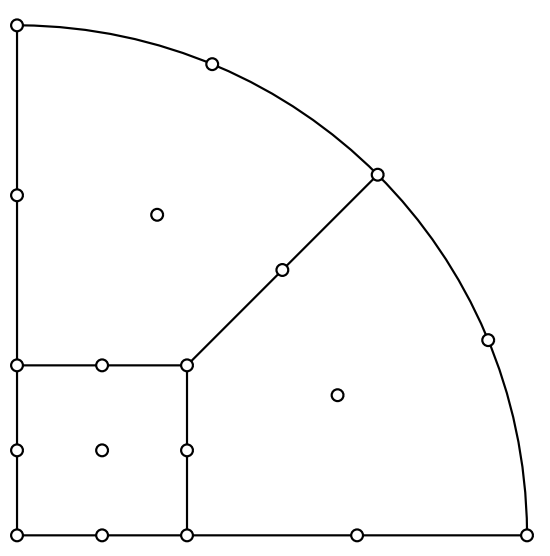

(a) $p=2$

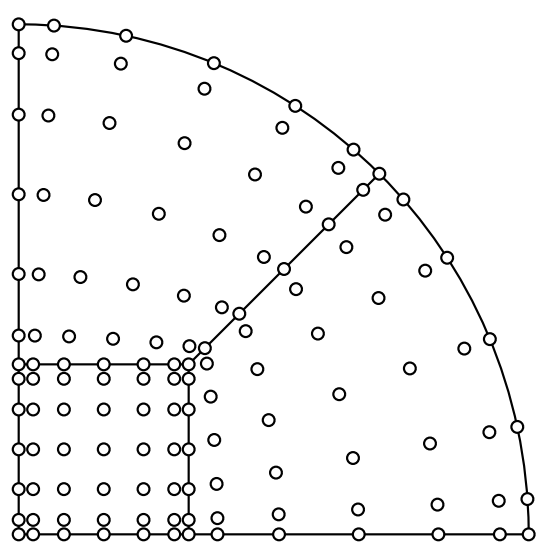

(b) $p=6$

Figure 1: Representative LSFE elements and nodes for the upper right quadrant of the full circular-plate system ( 3 of 12 elements shown) for (a) $p=2$ and (b) $p=6$ refinement.

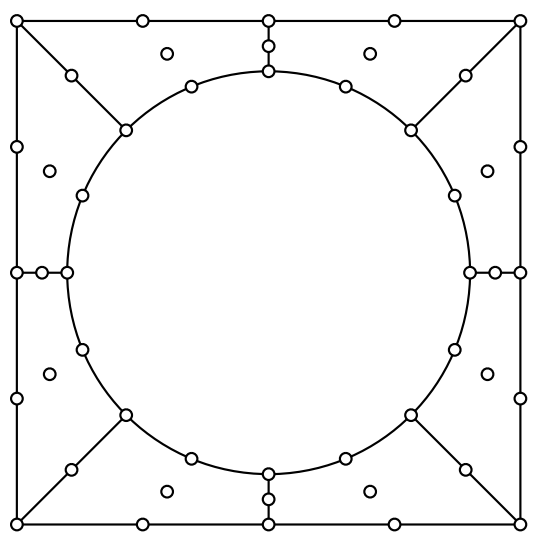

Figure 2: Representative LSFE elements and nodes for the upper right quadrant of the full perforated-plate system ( 8 of 32 elements shown) for $p=2$ refinement. 
the same as those described above. Table 1 shows grid-independent nondimensional center transverse-displacement solutions, $\left(\frac{w_{c} h^{3} E_{2}}{q_{0} a^{4}}\right) \times 10^{3}$, where $w_{c}$ is center displacement. Converged results were obtained with increasing $p$ in the LSFE model and increasing number of elements in the SHELL281 model. The ANSYS and LSFE results agree with those of Reddy [22].

Table 1: Nondimensional center transverse displacement of a square composite plate under uniform load.

\begin{tabular}{|l|l|}
\hline Reddy [22] & 19.469 \\
ANSYS, SHELL281 & 19.4687 \\
LSFE & 19.4687 \\
\hline
\end{tabular}

\subsection{Static deformation of a clamped circular plate}

We consider here the static deformation of a clamped circular plate under uniform load with magnitude $q_{0}$. Figure 3 shows the benchmark nondimensional in-plane and transverse midplane displacements

$$
\left\{\bar{u}^{b}, \bar{v}^{b}, \bar{w}^{b}\right\}=\frac{h^{3} E_{2}}{q_{0} R^{4}}\left\{u^{b}, v^{b}, w^{b}\right\}
$$

along $y=0$ and $x=0$ for plates with $h / R=10$ and $h / R=100$. These benchmark displacements were calculated with a highly refined LSFE model $(p=20)$. In-plane displacements are activated due to orthotropic nature of the composite materials, and the in-plane displacements are much larger in the thicker-plate configuration.

Figure 4 shows the normalized error of the center transverse displacement $w_{c}$ (compared with the benchmark center transverse displacement $w_{c}^{b}$ ) as a function of the total number of model nodes $n$. Errors are shown for ANSYS SHELL281-element models and LSFE models. For the LSFE solutions, exponential convergence (with increasing model size via increasing element order) to machine precision is observed. Similar accuracy is observed for both plate thicknesses. The FE results (ANSYS SHELL281) show algebraic convergence with model refinement. For the three coarsest FE models, the ANSYS solutions for the thinner plate $(h / R=100)$ are significantly less accurate than those for $h / R=10$. For the $h / R=10$ plate, LSFEs offer 


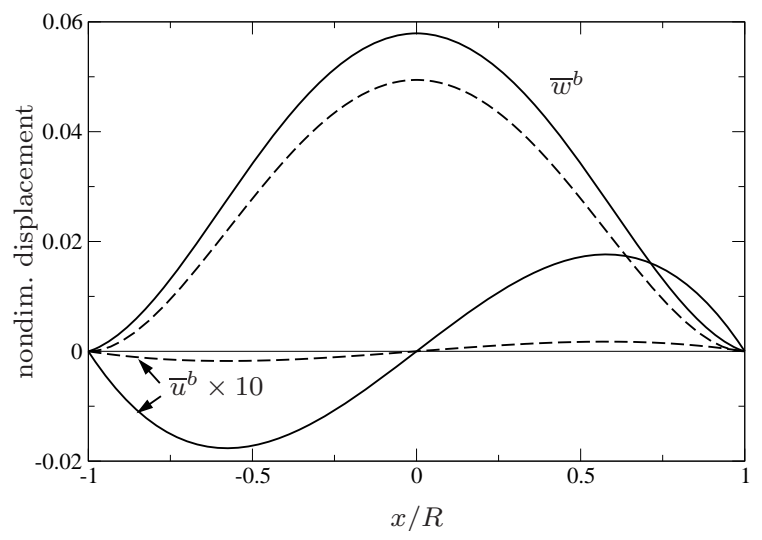

(a) $y=0$

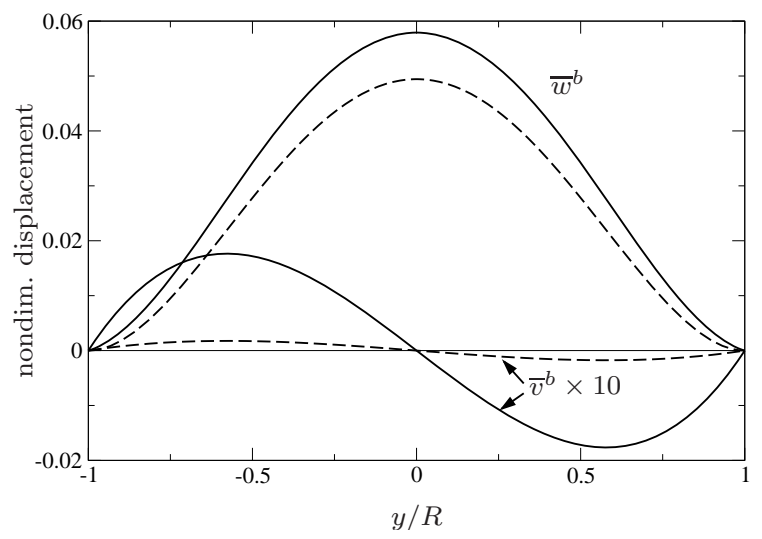

(b) $x=0$

Figure 3: Nondimensional transverse and in-plane benchmark displacement profiles of the circular plate at (a) $y=0$ (where $\bar{v}^{b}=0$ ) and (b) $x=0\left(\right.$ where $\left.\bar{u}^{b}=0\right)$ for $h / R=10$ (solid lines) and $h / R=100$ (dashed lines). 
comparable accuracy with the FEs for the two coarsest meshes, but otherwise provide orders of magnitude better accuracy for a given model size. For the thinner plate, LSFEs provide orders of magnitude more accuracy for all model sizes examined.

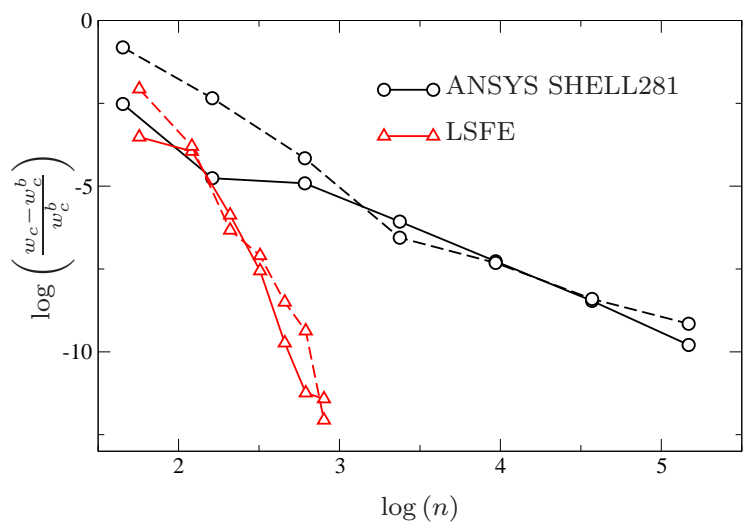

Figure 4: Normalized error of the circular-plate center displacement as a function of the number of model nodes $n$. Results are shown for $h / R=10$ (solid lines) and $h / R=100$ (dashed lines). LSFE results are shown for $p=2,3, \ldots, 8$.

Figure 5(a) shows the normalized center-displacement errors of Fig. 4 plotted against the computation time taken by the HSL_MA97 sparse linear solver. As described above, computation time was taken as the sum of matrix factorization and solve time. Figure 5(b) shows the same data plotted against the memory required. Memory required was calculated based on the number non-zero sparse storage entries and indexing data and the number of entries in the solution and right-hand-side vectors. The superior performance of LSFEs compared to the SHELL281 elements follows a similar trend as that shown in Fig. 4. For the thicker plate, LSFEs show comparable performance with the FEs for the two least-refined meshes, but otherwise provide orders of magnitude better accuracy for a given computation time or for a given memory-storage size. For the thinner plate, LSFEs dramatically outperform the SHELL281 elements at all refinement levels.

The SHELL281-element data shown in Figs. 4-5 imply a degradation in model performance for $h / R \rightarrow 0$ for a fixed model size (at the lower end of refinement). Figure 6 shows the normalized error of the center displacement for four $h / R$ values. The LSFE model had 209 nodes $(p=4)$ and the SHELL281-element model had 609 nodes. These refinement levels were 


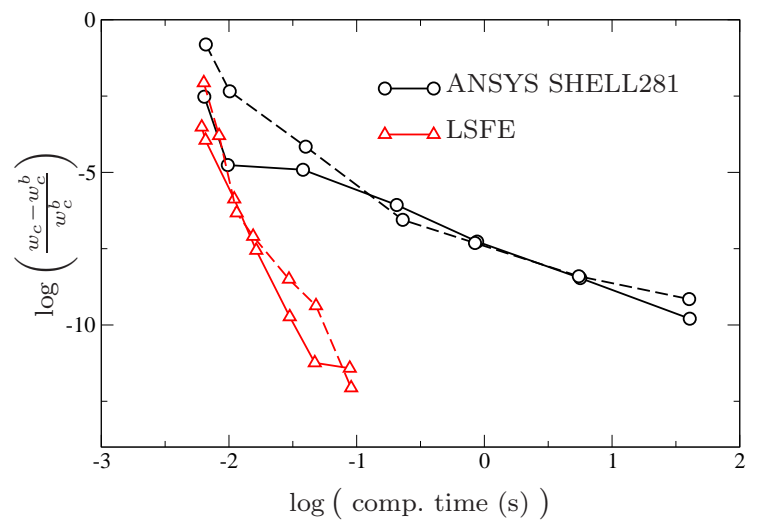

(a)

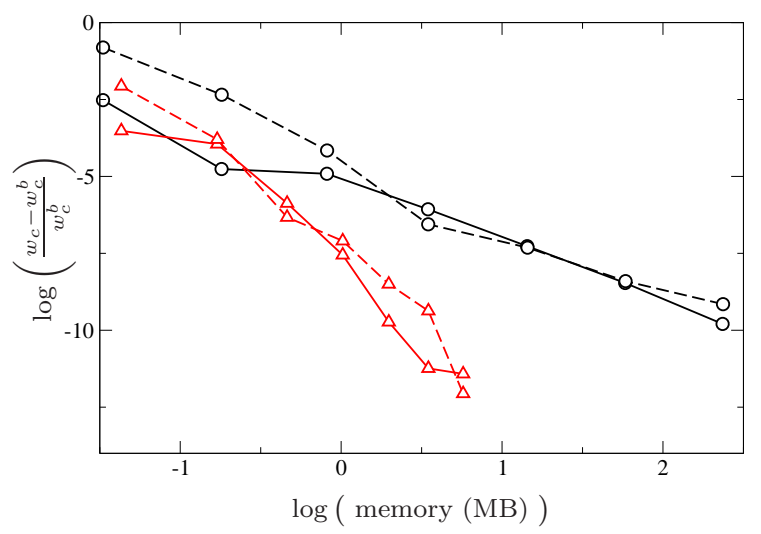

(b)

Figure 5: Normalized error of the circular-plate center displacement as a function of (a) computation time and (b) required computation memory. Results are shown for $h / R=10$ (solid lines) and $h / R=100$ (dashed lines). LSFE results are shown for $p=2,3, \ldots, 8$. 
chosen because they yielded nominally equivalent center-displacement accuracy for the thickest-plate configuration $(h / R=10)$. For each of these $h / R$ configurations, the benchmark solution was calculated with a highly refined $(p=20)$ LSFE model. The center displacements calculated with SHELL281 elements show significant accuracy degradation with decreasing $h / R$. LSFE results are shown where the stiffness matrices were calculated with either mixed-reduced quadrature (as described above) or full $(p+1) \times(p+1)$ GaussLegendre quadrature. In all cases, the LSFE results are dramatically more accurate than those of the SHELL281 elements. The LSFE results calculated with mixed-reduced quadrature are insensitive to changes in thickness, whereas the LSFEs calculated with full quadrature show a degradation in performance with decreasing plate thickness.

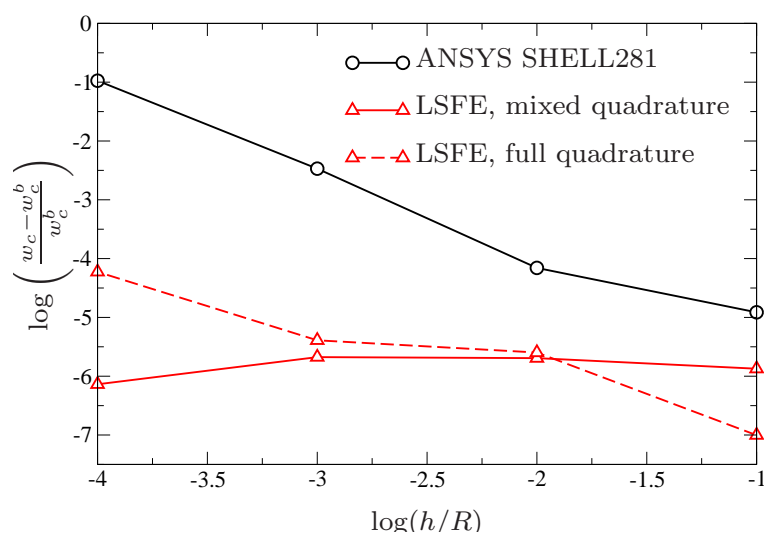

Figure 6: Normalized error of the circular-plate center displacement as a function of $h / R$. The LSFE model had 209 nodes (12 elements with $p=4)$ and the ANSYS SHELL281element model had 609 nodes (192 elements).

The results shown above were calculated with a sparse direct linear solver, which is appropriate considering the small sizes of the models at hand. However, we expect that for a large enough model, direct solution will be expensive, and one would resort to an iterative solver. Iterative solution methods are also of interest due to their ease of parallelization for simulation on multiple computer cores. Hence, we consider performance of several iterative-solve configurations for LSFE models.

Figure 7 shows computation times as a function of LSFE model size (number of nodes) when calculated under three solver configurations and for two plate thicknesses $(h / R=10$ and 100). LSFE models were calculated with 
$p=2,3, \ldots, 10,12,14$. First shown are the computation times for sparse direct solves. Second shown are computation times for Jacobi-preconditioned CG (JPCG) solves where matrix-vector products were calculated with a matrix-free algorithm that exploits a tensor-product factorization for the matrix-vector product. The third are computation times for JPCG for which the global stiffness matrices were stored sparsely, and matrix-vector products exploited sparsity. For all but the smallest two models, sparse direct solution is the most expedient. For the smallest models, it is likely that the computation overhead associated with factorization and linear-system solution in the direct solver is dominating computation time, whereas there is no such overhead in the JPCG solution. While the computation times for the two plate thicknesses are essentially the same for direct solution, the thinner plate requires more JPCG computation time than the thicker plate, which implies a larger condition number. As described above, matrix-free evaluation of matrix-vector products is accomplished in a factorized form, which requires $O\left(p^{3}\right)$ operations, whereas sparse matrix-vector products require $O\left(p^{4}\right)$ operations. These two different operation requirements are reflected by the different JPCG slopes shown in Fig. 7. While JPCG solution with matrixfree evaluation of matrix-vector products will be more efficient than sparse matrix-vector products for sufficiently large $p$, we see that the cross-over is at $p=8$ for the systems at hand.

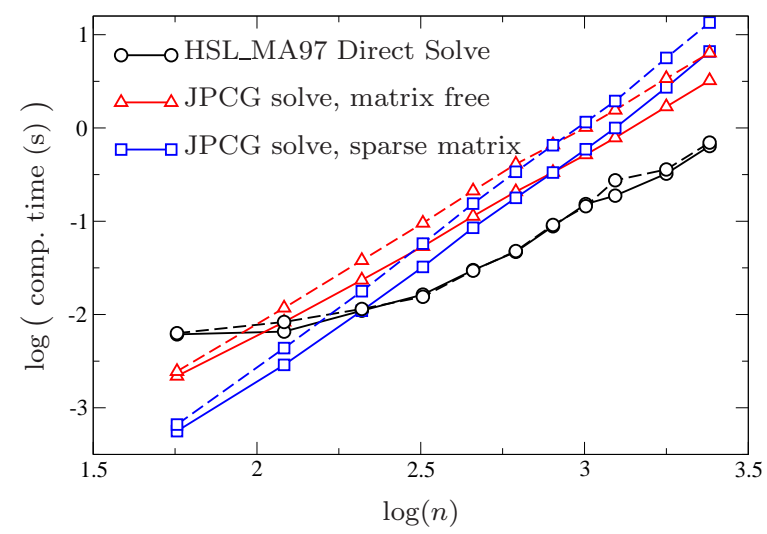

Figure 7: LSFE computation times as for the circular plate a function of the total number of nodes. Data are shown for the $h / R=10$ (solid line) and 100 (dashed line) configurations. LSFE results are shown for $p=2, \ldots, 9,10,12,14$. 


\subsection{Static deformation of a corner-pinned square plate}

We consider the deformation of a composite corner-supported square plate with $a / h=10$ under a uniform load. This is a common test case for spurious zero energy modes (see, e.g., [23]). A benchmark solution was calculated with an extremely refined ANSYS SHELL281-element model on a structured grid with about $1.3 \times 10^{7}$ nodes. The LSFE model was composed of 4 square elements and stiffness matrices were calculated with full quadrature. As perhaps expected, the stiffness matrix was singular when calculated with mixed-reduced quadrature. LSFE Figure 8 shows the normalized error of the center displacement as a function of the total number of nodes. The coarsest SHELL281 and LSFE models had 65 and 81 nodes, respectively. For this configuration, both the SHELL281 and LSFE models show poor convergence rates. However, the LSFE results are significantly more accurate for a given model size.

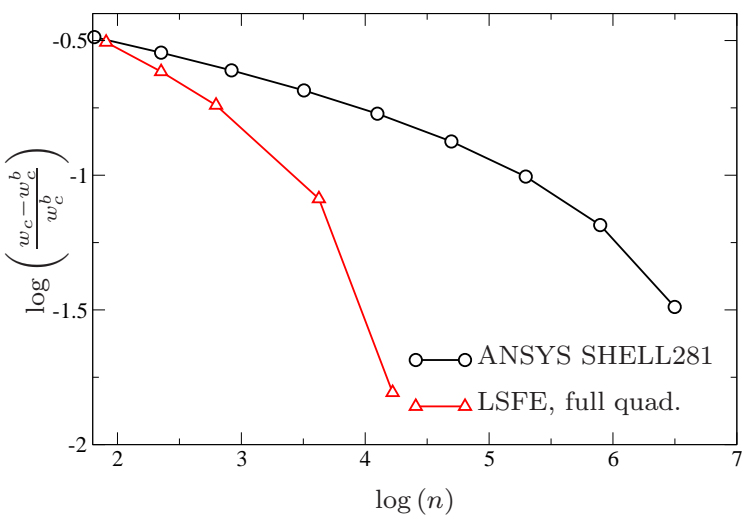

Figure 8: Normalized error of center displacement of a corner-supported square plate as a function of the number of model nodes $n$. Results are shown for $h / R=10$. LSFE results are shown for $p=4,7,12,32,64$.

\subsection{Static deformation of a clamped perforated square plate}

As a final static study, we consider the deformation of a clamped-edge perforated square plate with $a / h=20$ under a uniform pressure load. The benchmark solution was calculated with a refined ANSYS SHELL281 model with $1.6 \times 10^{6}$ nodes. Figure 9 shows normalized center-displacement errors as a function of the total number of nodes and as a function of computation time. LSFE results were calculated with both mixed-reduced and full quadrature. 
Here, LSFE results show little dependence on quadrature choice. While LSFE results show better convergence in with model size when compared with the SHELL281 results, we see that both LSFE and SHELL281 exhibit similar performance in terms of computation time.

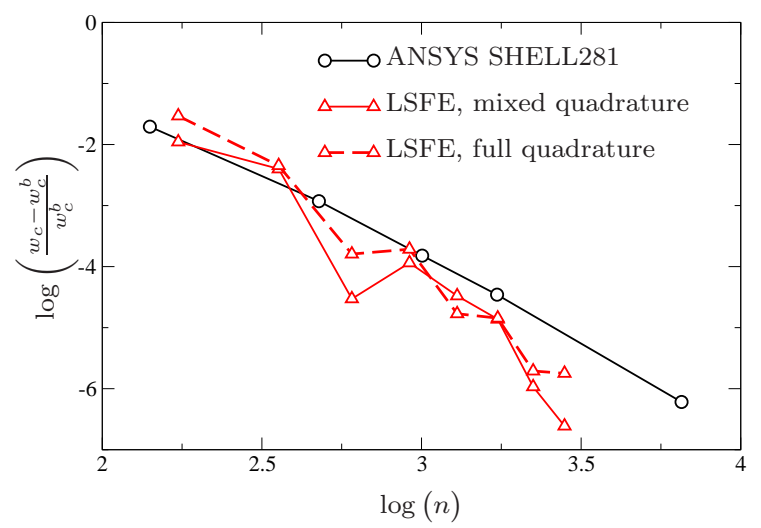

(a)

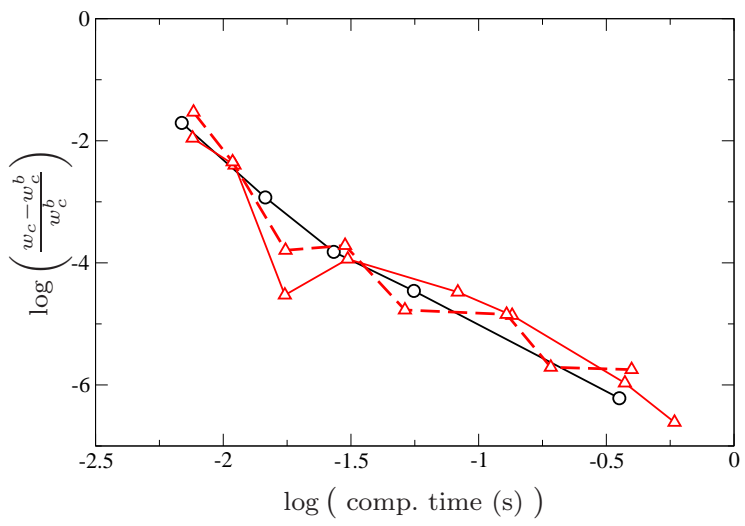

(b)

Figure 9: Normalized error of center displacement of a clamped-edge perforated square plate as a function of (a) the number of nodes and (b) computation time. LSFE results are shown for $p=2,3, \ldots, 9$.

\subsection{Dynamic deformation of a circular plate}

We consider here the performance of LSFEs and ANSYS SHELL281 elements under implicit direct time integration with fixed time increment $\Delta t$. For the SHELL281 elements, we examine solution accuracy for both consistent and lumped mass matrices. LSFE mass matrices are lumped through 
nodal quadrature. Time integration is accomplished with a standard Trapezoidal rule algorithm. The linear-system solve required at each step was accomplished with the HSL_MA97 sparse direct solver. As in the staticdeformation studies, ANSYS and LSFE stiffness and mass matrices were imported into our time-integration software.

We consider the circular plate described above for $h / R=10$ and 100 under a uniform transverse pressure load given by

$$
p(\bar{t})= \begin{cases}\frac{q_{0}}{2}[1-\cos (10 \bar{t} \pi)], & 0 \leq \bar{t} \leq 0.1, \\ q_{0}, & \bar{t}>0.1,\end{cases}
$$

where $\bar{t}$ is nondimensional time given by

$$
\bar{t}=\frac{t h}{R^{2}} \sqrt{\frac{E_{2}}{\rho}}
$$

and where $t$ is dimensional time and $\rho$ is density. Figure 10 shows the benchmark nondimensional center-displacement histories $\bar{w}_{c}^{b}$ as a function of nondimensional time. Benchmark solutions were calculated with a highly refined LSFE model with $p=20$ and a nondimensional time increment $\Delta \bar{t}=5 \times 10^{-6}$.

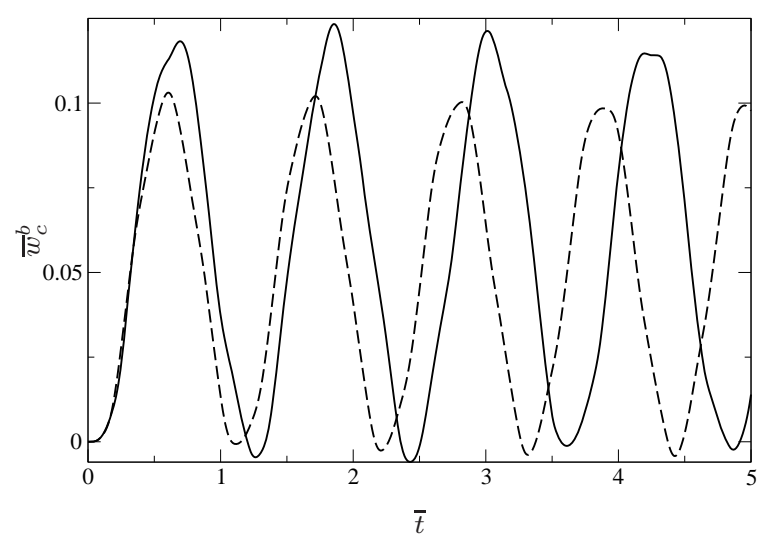

Figure 10: Nondimensional benchmark transverse center displacement histories for cicular plates with $h / R=10$ (solid line) and 100 (dashed line) and for a uniform pressure load described by Eq. (10). Results were calculated with a refined LSFE model.

Figure 11 shows the normalized maximum error of center displacement $\epsilon_{\max }$ as a function of nondimensional time increment, where

$$
\epsilon_{\max }=\frac{\left\|w_{c}-w_{c}^{b}\right\|_{\max }}{\left\|w_{c}^{b}\right\|_{\max }}
$$


and where $\|\cdot\|_{\max }$ denotes the maximum norm calculated over $0 \leq \bar{t} \leq 5$. Results for SHELL281 models are shown for $n=162$ and 609 nodes where the mass matrices were consistently cacluated. Alsos shown are SHELL281 results for a lumped matrix and $n=609$. Results for LSFE models are shown for $n=209$ and 617 nodes. We make several observations regarding these results. First, we see that as $\Delta t \rightarrow 0$ errors approach a constant, where the error is dominated by spatial-discretization errors. This plateau error level decreases with increasing spatial refinement. Second, these results show that, for approximately the same model size, an LSFE model provides access to significantly more accurate solutions than the SHELL281 elements as $\Delta \bar{t} \rightarrow$ 0 . For example, for $n \approx 600$ and $\Delta \bar{t}=10^{-3}$, the LSFE results provided 20 times and 34 times less error for $h / R=10$ and 100, respectively. The SHELL281 results for $n=609$ and a lumped mass matrix are significantly worse than those calculated with a consistent mass matrix.

Figure 12 shows the normalized maximum center-displacement errors of of Fig. 11 plotted as a function of computation time. For a given $\Delta \bar{t}$ and nominally equivalent model size, we see that an LSFE model takes slightly longer to calculate, which can be attributed to the fuller matrices in the LSFE systems. For example, for $\Delta \bar{t}=10^{-2}$ the SHELL281 $n=609$ model and the LSFE $n=617$ model require $6.7 \mathrm{~s}$ and $7.7 \mathrm{~s}$, respectively. LSFEs, at their worst, require approximately the same computation time as the SHELL 281 elements for a given accuracy level. However, at their best, LSFEs provide more than an order of magnitude increase in accuracy for a given computation time over their SHELL281-element counterparts.

\section{Concluding remarks}

We examined the performance of Legendre spectral finite elements with respect to that of ANSYS SHELL281 serendipity-type elements for several two-layer composite plates. In our comparisons, both model types were solved with the same static- or dynamic-solver algorithms and software. LSFE solutions were examined where the stiffness matrix was calculated with either a mixed reduced quadrature, which was shown to be locking free for isotropic RM plates by Brito and Sprague [5], or full quadrature. For the dynamic simulations, LSFE mass matrices were lumped through the use of nodal quadra-

ture. Solutions for SHELL281 elements were examined with lumped and consistent mass matrices. 


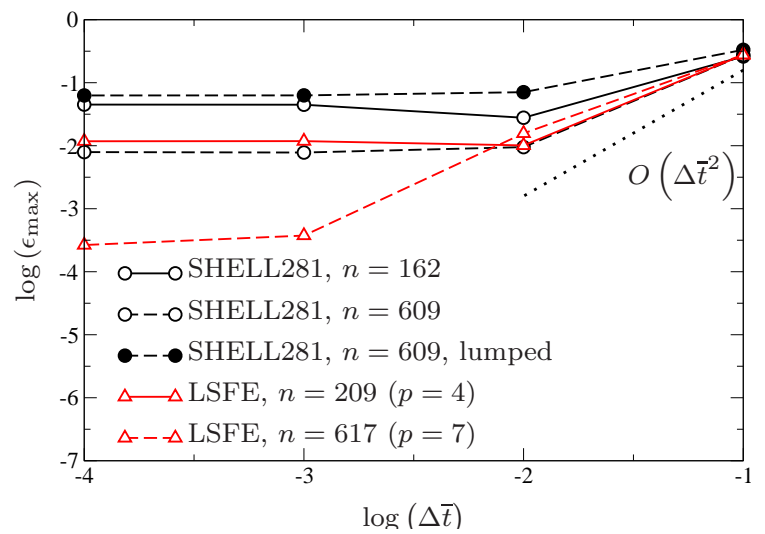

(a) $h / R=10$

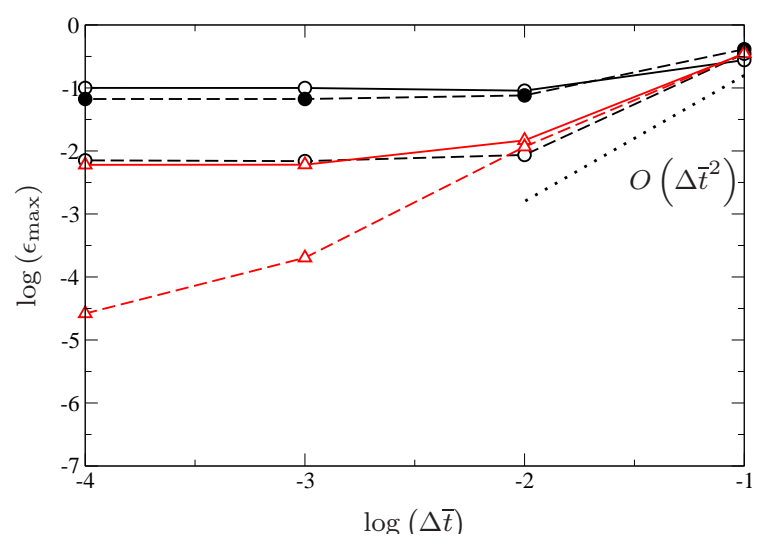

(b) $h / R=100$

Figure 11: Normalized maximum error of the cicular-plate center displacment calculated over $0 \leq \bar{t} \leq 5$ as a function of nondimensional time increment. The dotted lines illustrate second-order convergence. 


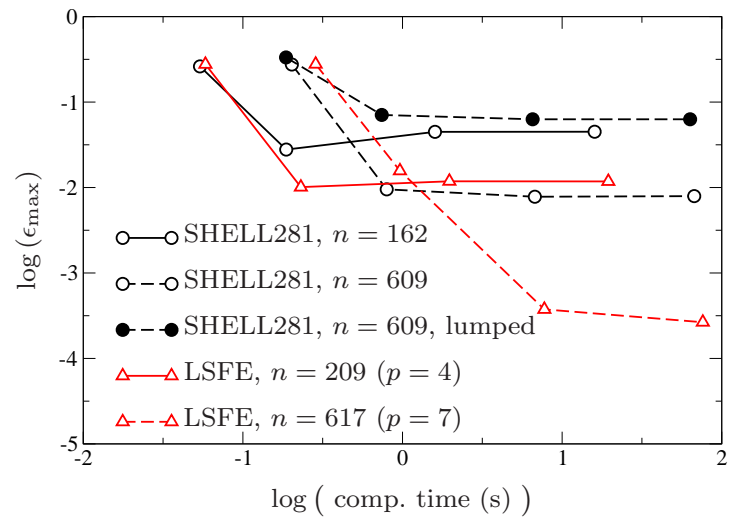

(a) $h / R=10$

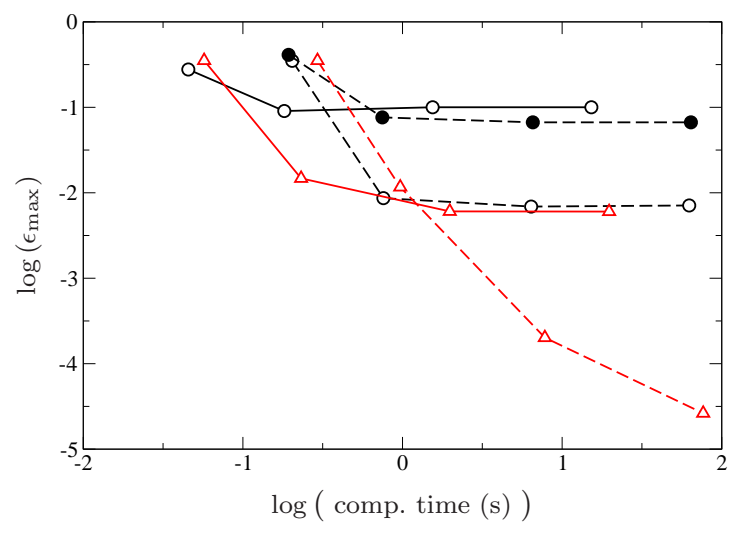

(b) $h / R=100$

Figure 12: Normalized maximum error of Fig. 11 plotted as a function of computation time. 
For the static-deformation studies of a clamped-edge circular plate, the LSFEs greatly outperformed the SHELL281 elements in all aspects examined. In particular, compared to SHELL281-element models, LSFEs can provide dramatically more accuracy for a given number of model nodes, for a given computation time, and for a given computer memory storage. Further, for this case, the LSFEs were shown to be much more robust for thin plates when calculated with mixed reduced quadrature. However, LSFEs calculated with full Gauss-Legendre quadrature showed a marginal degradation in accuracy with decreasing plate thickness. The degradation was also shown for the SHELL281-element model. We also examined static response of a corner-supported composite square plate. For this case, the LSFE stiffness matrices calculated with mixed-reduced quadrature were rank deficient. However, stiffness matrices calculated with full-quadrature yielded solutions more accurate than those produced with SHELL281 elements. In our final static study, we examined a clamped-edge square perforated plate where LSFE elements were highly deformed. Here, SHELL281 and LSFE solutions had accuracy that was commensurate in terms of model size and computation time.

While sparse direct solution of the relatively small linear systems examined here was most efficient, we examined the performance of Jacobipreconditioned CG iterative solution of LSFE systems, where matrix-vector products were evaluated with a sparsely stored stiffness matrix or a matrixfree tensor-product-factorized form. For these systems, LSFEs with $p<8$ were most efficiently solved with sparse storage of the stiffness matrix, while LSFEs with $p>8$ were most efficiently solved with the matrix-free form. However, the matrix-free form is appealing at all refinement levels because matrix-vector-product evaluation requires minimal memory storage and is readily parallelized.

We have shown that LSFEs can offer orders of magnitude more accuracy, for a given model size or computation time, over the ANSYS SHELL281 elements for static and dynamic deformation of Reissner-Mindlin composite plates. LSFE solutions, with stiffness matrices calculated with mixed reduced quadrature, were insensitive to shear locking, but can be susceptible to spurious zero-energy modes. Finally, while we focused here on implicit time integration, the diagonal mass matrices produced through nodal quadrature make LSFEs well suited to explicit time integration. Looking forward, we hope to extend this work in the creation of shell elements for linear and geometrically nonlinear deformation. We will build upon our success in applying 
the Legendre spectral element method to geometrically exact beam theory $[24,25]$ for large elastic deformations.

\section{Acknowledgment}

This work was supported by the U.S. Department of Energy under Contract No. DE-AC36-08-GO28308 with the National Renewable Energy Laboratory. Funding was provided by the NREL Laboratory Directed Research and Development (LDRD) program through the grant "High-Fidelity Computational Modeling of Wind-Turbine Structural Dynamics." The research was performed using computational resources sponsored by the Department of Energy's Office of Energy Efficiency and Renewable Energy and located at the National Renewable Energy Laboratory. We acknowledge insightful comments from the reviewers that led to an improved paper.

\section{Appendix A. Mass and stiffness matrices}

We describe here the LSFE basis functions, and the resulting mass and stiffness matrices used in our calculations for composite Reissner-Mindlin plates. Mass and stiffness matrices described here follow the standard form described in Ochoa and Reddy [18]. As described in Section 2.1, LSFE twodimensional basis functions are a tensor product of $p^{\text {th }}$-order one-dimensional Lagrangian interpolant functions with nodes located at the $(p+1)$ GLL points. The basis functions introduced in Eq. (3) may be written for the reference element domain $\Omega_{\square}=\{(\xi, \eta) \mid-1 \leq \xi \leq 1,-1 \leq \eta \leq 1\}$ as

$$
\begin{aligned}
& \Phi_{\widehat{i}}(\xi, \eta)=\phi_{i}(\xi) \phi_{j}(\eta), \\
& \Phi_{\widehat{\ell}}(\xi, \eta)=\phi_{\ell}(\xi) \phi_{m}(\eta),
\end{aligned}
$$

where $\widehat{i}, \widehat{\ell} \in\left\{1, \ldots,(p+1)^{2}\right\}$ are defined in lexicographical order as

$$
\begin{aligned}
& \widehat{i}=i+(p+1)(j-1), \\
& \widehat{\ell}=\ell+(p+1)(m-1),
\end{aligned}
$$

for $i, j, \ell, m \in\{1, \ldots, p+1\}$. The one-dimensional Lagrangian interpolants are defined for $-1 \leq \zeta \leq 1$ as (see, e.g., Deville et al. [26])

$$
\phi_{i}(\zeta)= \begin{cases}1, & \text { for } \zeta=\zeta_{i}, \\ -\frac{\left(1-\zeta^{2}\right)}{p(p+1) L_{p}\left(\zeta_{i}\right)\left(\zeta-\zeta_{i}\right)} \frac{\mathrm{d} L_{p}}{\mathrm{~d} \zeta}, & \text { for } \zeta \neq \zeta_{i},\end{cases}
$$


where $L_{p}(\zeta)$ is the Legendre polynomial of degree $p$ and $\zeta_{i}$ is the location of the $i^{t h}$ GLL point. The $p+1$ GLL points are the zeros of $\left(1-\zeta^{2}\right) \frac{\mathrm{d} L_{p}(\zeta)}{\mathrm{d} \zeta}$ in $-1 \leq \zeta \leq 1$.

The element-level mass matrix is given by

$$
\mathbf{M}=\left[\begin{array}{ccc}
\mathbf{M}_{1,1}^{d} & \cdots & \mathbf{M}_{1,(p+1)^{2}}^{d} \\
& \ddots & \vdots \\
\text { sym. } & & \mathbf{M}_{(p+1)^{2},(p+1)^{2}}^{d}
\end{array}\right]
$$

where

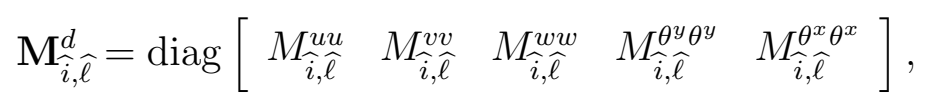

and

$$
\begin{aligned}
& M_{\widehat{i}, \widehat{\ell}}^{u u}=M_{\widehat{i}, \widehat{\ell}}^{v v}=M_{\widehat{i}, \widehat{\ell}}^{w w}=\int_{\Omega_{\square}} \rho h \phi_{i}(\xi) \phi_{j}(\eta) \phi_{\ell}(\xi) \phi_{m}(\eta) J \mathrm{~d} \xi \mathrm{d} \eta \\
& M_{\widehat{i}, \widehat{\ell}}^{\theta^{y} \theta^{y}}=M_{\widehat{i}, \widehat{\ell}}^{\theta^{x} \theta^{x}}=\frac{1}{12} \int_{\Omega_{\square}} \rho h^{3} \phi_{i}(\xi) \phi_{j}(\eta) \phi_{\ell}(\xi) \phi_{m}(\eta) J \mathrm{~d} \xi \mathrm{d} \eta
\end{aligned}
$$

for $\widehat{i}, \widehat{\ell} \in\left\{1, \ldots,(p+1)^{2}\right\}$, and where $J$ is the determinant of the mapping Jacobian. When $(p+1) \times(p+1)$ GLL quadrature is employed, $\mathbf{M}$ is diagonal.

The element-level stiffness matrix is given by

$$
\mathbf{K}=\left[\begin{array}{ccc}
\mathbf{K}_{1,1} & \cdots & \mathbf{K}_{1,(p+1)^{2}} \\
& \ddots & \vdots \\
\text { sym. } & & \mathbf{K}_{(p+1)^{2},(p+1)^{2}}
\end{array}\right],
$$

where

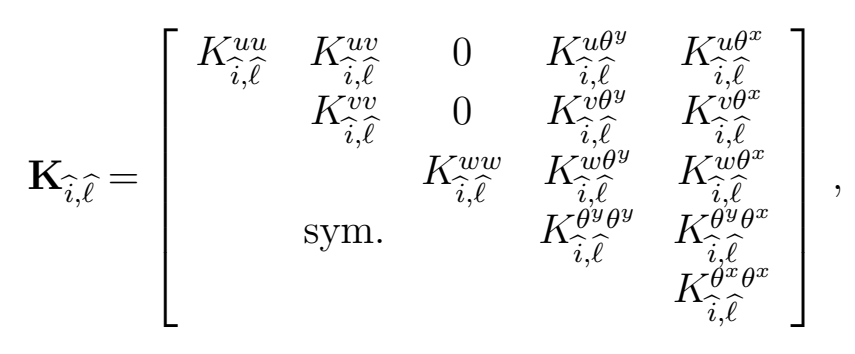


for $\widehat{i}, \widehat{\ell} \in\left\{1, \ldots,(p+1)^{2}\right\}$. Each component of Eq. (A.10) may be written as a combination of three different stiffness inner-product forms:

$$
\begin{aligned}
& K_{i, \hat{\ell}}^{u u}=K_{\widehat{i}, \widehat{\ell}}^{1}\left(A_{11}, A_{16}, A_{16}, A_{66}\right), \\
& K_{\hat{i}, \widehat{\ell}}^{u v}=K_{\widehat{i}, \widehat{\ell}}^{1}\left(A_{16}, A_{12}, A_{66}, A_{26}\right), \\
& K_{\hat{i}, \widehat{\ell}}^{u \theta^{y}}=K_{\hat{i}, \widehat{\ell}}^{1}\left(B_{11}, B_{16}, B_{16}, B_{66}\right), \\
& K_{\widehat{i}, \hat{\ell}}^{u \theta^{x}}=K_{\hat{i}, \widehat{\ell}}^{1}\left(B_{16}, B_{12}, B_{66}, B_{26}\right) \text {, } \\
& K_{\widehat{i}, \widehat{\ell}}^{v v}=K_{\hat{i}, \widehat{\ell}}^{1}\left(A_{66}, A_{26}, A_{26}, A_{22}\right), \\
& K_{\widehat{i}, \widehat{\ell}}^{v \theta^{y}}=K_{\hat{i}, \widehat{\ell}}^{1}\left(B_{16}, B_{66}, B_{12}, B_{26}\right), \\
& K_{\widehat{i}, \hat{\ell}}^{v \theta^{x}}=K_{\hat{i}, \widehat{\ell}}^{1}\left(B_{66}, B_{26}, B_{26}, B_{22}\right) \text {, } \\
& K_{\widehat{i}, \hat{\ell}}^{w w}=K_{\widehat{i}, \widehat{\ell}}^{1}\left(A_{55}, A_{45}, A_{45}, A_{44}\right) \text {, } \\
& K_{\widehat{i}, \widehat{\ell}}^{w \theta^{y}}=K_{\widehat{i}, \widehat{\ell}}^{2}\left(A_{55}, A_{45}\right), \\
& K_{\hat{i}, \hat{\ell}}^{w \theta^{x}}=K_{\hat{i}, \hat{\ell}}^{2}\left(A_{45}, A_{44}\right), \\
& K_{\widehat{i}, \widehat{\ell}}^{\theta^{y} \theta^{y}}=K_{\widehat{i}, \widehat{\ell}}^{1}\left(D_{11}, D_{16}, D_{16}, D_{66}\right)+K_{\hat{i}, \widehat{\ell}}^{3}\left(A_{55}\right) \text {, } \\
& K_{\widehat{i}, \widehat{\ell}}^{\theta^{y} \theta^{x}}=K_{\widehat{i}, \widehat{\ell}}^{1}\left(D_{16}, D_{12}, D_{66}, D_{26}\right)+K_{\hat{i}, \widehat{\ell}}^{3}\left(A_{45}\right) \text {, } \\
& K_{\hat{i}, \hat{\ell}}^{\theta^{x} \theta^{x}}=K_{\widehat{i}, \widehat{\ell}}^{1}\left(D_{66}, D_{26}, D_{26}, D_{22}\right)+K_{\widehat{i}, \hat{\ell}}^{3}\left(A_{44}\right) \text {, }
\end{aligned}
$$

where $A_{i j}, B_{i j}$, and $D_{i j}$ (for $\left.(i j) \in\{11,12,22,16,26,66\}\right)$ are extensional stiffnesses, bending-extensional-coupling stiffnesses, and bending stiffnesses, respectively, which may be found in any number of textbooks (see, e.g., [18]), and

$$
\begin{aligned}
K_{\hat{i}, \hat{\ell}}^{1}(a, b, c, d)=\int_{\Omega_{\square}}\{ & \left(\frac{\partial \phi_{i}}{\partial \xi} \phi_{j} \frac{\partial \xi}{\partial x}+\phi_{i} \frac{\partial \phi_{j}}{\partial \eta} \frac{\partial \eta}{\partial x}\right)\left[a\left(\frac{\partial \phi_{\ell}}{\partial \xi} \phi_{m} \frac{\partial \xi}{\partial x}+\phi_{\ell} \frac{\partial \phi_{m}}{\partial \eta} \frac{\partial \eta}{\partial x}\right)\right. \\
& \left.+b\left(\frac{\partial \phi_{\ell}}{\partial \xi} \phi_{m} \frac{\partial \xi}{\partial y}+\phi_{\ell} \frac{\partial \phi_{m}}{\partial \eta} \frac{\partial \eta}{\partial y}\right)\right] \\
& +\left(\frac{\partial \phi_{i}}{\partial \xi} \phi_{j} \frac{\partial \xi}{\partial y}+\phi_{i} \frac{\partial \phi_{j}}{\partial \eta} \frac{\partial \eta}{\partial y}\right)\left[c\left(\frac{\partial \phi_{\ell}}{\partial \xi} \phi_{m} \frac{\partial \xi}{\partial x}+\phi_{\ell} \frac{\partial \phi_{m}}{\partial \eta} \frac{\partial \eta}{\partial x}\right)\right. \\
& \left.\left.+d\left(\frac{\partial \phi_{\ell}}{\partial \xi} \phi_{m} \frac{\partial \xi}{\partial x}+\phi_{\ell} \frac{\partial \phi_{m}}{\partial \eta} \frac{\partial \eta}{\partial x}\right)\right]\right\} J \mathrm{~d} \xi \mathrm{d} \eta, \quad \text { (A.24) }
\end{aligned}
$$




$$
\begin{aligned}
& K_{\hat{i}, \hat{\ell}}^{2}(a, b)=\int_{\Omega_{\square}} {\left[a\left(\frac{\partial \phi_{i}}{\partial \xi} \phi_{j} \frac{\partial \xi}{\partial x}+\phi_{i} \frac{\partial \phi_{j}}{\partial \eta} \frac{\partial \eta}{\partial x}\right)\right.} \\
&\left.+b\left(\frac{\partial \phi_{i}}{\partial \xi} \phi_{j} \frac{\partial \xi}{\partial y}+\phi_{i} \frac{\partial \phi_{j}}{\partial \eta} \frac{\partial \eta}{\partial y}\right)\right] \phi_{\ell} \phi_{m} J \mathrm{~d} \xi \mathrm{d} \eta \\
& K_{\overparen{i}, \hat{\ell}}^{3}(a)=\int_{\Omega_{\square}} a \phi_{i} \phi_{j} \phi_{\ell} \phi_{m} J \mathrm{~d} \xi \mathrm{d} \eta .
\end{aligned}
$$

\section{Appendix B. Tensor-product factorization}

We consider here matrix-vector products involving the element-level generalized displacement vector and stiffness matrix, i.e.,

$$
\mathbf{B}=\mathbf{K U}
$$

where $\mathbf{B}=\left[\begin{array}{llll}\mathbf{B}_{1} & \ldots & \mathbf{B}_{(p+1)^{2}}\end{array}\right]^{T}$ and $\mathbf{B}_{\hat{i}}=\left[\begin{array}{lllll}B_{\hat{i}}^{u} & B_{\hat{i}}^{v} & B_{\hat{i}}^{w} & B_{\hat{i}}^{\theta^{y}} & B_{\hat{i}}^{\theta^{x}}\end{array}\right]^{T}$. With $\mathbf{K}$ calculated, this product requires on the order of $5(p+1)^{2} \times(p+1)^{2}$, i.e., $O\left(p^{4}\right)$, operations and $O\left(p^{4}\right)$ storage. Alternatively, one can write the matrix-vector product in a matrix-free form, where stiffness-matrix inner products are evaluated via quadrature on-the-fly, which requires $O\left(p^{6}\right)$ operations and negligible storage. Matrix-free matrix-vector products can, however, exploit a tensor-product factorization, which requires only $O\left(p^{3}\right)$ operations and $O\left(p^{2}\right)$ storage (for two-dimensional elements like plates and shells). The factorization is described in detail for isotropic plate elements in Brito and Sprague [5]. We describe here the final factorized form for composite plates, where, as in [5], we employed $(p+1) \times(p+1)$ nodal (GLL) quadrature for bending- and extensional-stiffness terms, and $p \times p$ Gauss-Legendre quadrature for shear-stiffness terms. In the factorization process, nodal quadrature provides significant savings through tensor contractions enabled by the orthogonality of Lagrangian interpolants in the nodal-quadrature discrete inner product.

We describe first the final expressions for terms associated with bending and extensional stiffness. The matrix-vector products for $B_{i}^{u}, B_{\hat{i}}^{v}, B_{\hat{i}}^{\theta^{y}}$, and 
$B_{\hat{i}}^{\theta^{x}}$ are as follows:

$$
\begin{aligned}
& B_{i j}^{u}=\sum_{p=1}^{p+1}\left\{D_{i p}\left[\gamma_{11 p j}\left(\psi_{p j}^{u, 1}+\psi_{p j}^{u, 2}\right)+\gamma_{21 p j}\left(\psi_{p j}^{u, 3}+\psi_{p j}^{u, 4}\right)\right] J_{p j} W_{p} W_{j}\right. \\
& \left.+D_{j p}\left[\gamma_{12 i p}\left(\psi_{i p}^{u, 1}+\psi_{i p}^{u, 2}\right)+\gamma_{22 i p}\left(\psi_{i p}^{u, 3}+\psi_{i p}^{u, 4}\right)\right] J_{i p} W_{i} W_{p}\right\} \text {, } \\
& B_{i j}^{v}=\sum_{p=1}^{p+1}\left\{D_{i p}\left[\gamma_{11 p j}\left(\psi_{p j}^{v, 1}+\psi_{p j}^{v, 2}\right)+\gamma_{21 p j}\left(\psi_{p j}^{v, 3}+\psi_{p j}^{v, 4}\right)\right] J_{p j} W_{p} W_{j}\right. \\
& \left.+D_{j p}\left[\gamma_{12 i p}\left(\psi_{i p}^{v, 1}+\psi_{i p}^{v, 2}\right)+\gamma_{22 i p}\left(\psi_{i p}^{v, 3}+\psi_{i p}^{v, 4}\right)\right] J_{i p} W_{i} W_{p}\right\}, \\
& \widetilde{b}_{i j}^{\theta^{y}}=\sum_{p=1}^{p+1}\left\{D_{i p}\left[\gamma_{11 p j}\left(\psi_{p j}^{\theta^{y}, 1}+\psi_{p j}^{\theta^{y}, 2}\right)+\gamma_{21 p j}\left(\psi_{p j}^{\theta^{y}, 3}+\psi_{p j}^{\theta^{y}, 4}\right)\right] J_{p j} W_{p} W_{j}\right. \\
& \left.+D_{j p}\left[\gamma_{12 i p}\left(\psi_{i p}^{\theta^{y}, 1}+\psi_{i p}^{\theta^{y}, 2}\right)+\gamma_{22 i p}\left(\psi_{i p}^{\theta^{y}, 3}+\psi_{i p}^{\theta^{y}, 4}\right)\right] J_{i p} W_{i} W_{p}\right\} \\
& +b_{i j}^{\theta^{y}, S}, \\
& \widetilde{b}_{i j}^{\theta^{x}}=\sum_{p=1}^{p+1}\left\{D_{i p}\left[\gamma_{11 p j}\left(\psi_{p j}^{\theta^{x}, 1}+\psi_{p j}^{\theta^{x}, 2}\right)+\gamma_{21 p j}\left(\psi_{p j}^{\theta^{x}, 3}+\psi_{p j}^{\theta^{x}, 4}\right)\right] J_{p j} W_{p} W_{j}\right. \\
& \left.+D_{j p}\left[\gamma_{12 i p}\left(\psi_{i p}^{\theta^{x}, 1}+\psi_{i p}^{\theta^{x}, 2}\right)+\gamma_{22 i p}\left(\psi_{i p}^{\theta^{x}, 3}+\psi_{i p}^{\theta^{x}, 4}\right)\right] J_{i p} W_{i} W_{p}\right\} \\
& +b_{i j}^{\theta^{x}, S},
\end{aligned}
$$

for all $i, j \in\{1, \ldots, p+1\}$, where $J_{i j}$ is the determinant of the mapping Jacobian evaluated at the $i j$ node (and GLL quadrature point), $W_{i}$ is the $i^{\text {th }}$ GLL quadrature weight, $\gamma_{i j \ell m}$ is the $i j$ entry of the inverse of the $2 \times 2$ Jacobian mapping matrix evaluated at the $\ell m$ node, and

$$
D_{i j}=\left.\frac{\mathrm{d} \phi_{i}}{\mathrm{~d} \zeta}\right|_{\zeta=\zeta_{j}},
$$


is the derivative of the $i^{\text {th }}$ Lagrangian interpolant evaluated at the $j^{\text {th }}$ GLL quadrature point. The $\psi_{i j}$ terms are intermediate tensors associated with bending and extensional stiffness (identified by superscripts) which are evaluated with $(p+1) \times(p+1)$ GLL (nodal) quadrature, which are listed below. The terms $b_{i j}^{\theta^{y}, S}$ and $b_{i j}^{\theta^{x}, S}$ are associated with shear stiffness, which are evaluated with $p \times p$ Gauss-Legendre quadrature and are described below.

The intermediate tensors in Eqs. (B.2)-(B.5) are evaluated in one of two forms dependent on material properties:

$$
\begin{aligned}
& \psi_{o p}^{u, 1}=\psi_{o p}^{1}\left(A_{11}, A_{16}, A_{12}, B_{11}, B_{16}, B_{12}\right) \text {, } \\
& \psi_{o p}^{u, 2}=\psi_{o p}^{2}\left(A_{11}, A_{16}, A_{12}, B_{11}, B_{16}, B_{12}\right) \text {, } \\
& \psi_{o p}^{u, 3}=\psi_{o p}^{1}\left(A_{16}, A_{66}, A_{26}, B_{16}, B_{66}, B_{26}\right) \text {, } \\
& \psi_{o p}^{u, 4}=\psi_{o p}^{2}\left(A_{16}, A_{66}, A_{26}, B_{16}, B_{66}, B_{26}\right) \\
& \psi_{o p}^{v, 1}=\psi_{o p}^{1}\left(A_{16}, A_{66}, A_{26}, B_{16}, B_{66}, B_{26}\right), \\
& \psi_{o p}^{v, 2}=\psi_{o p}^{2}\left(A_{16}, A_{66}, A_{26}, B_{16}, B_{66}, B_{26}\right) \\
& \psi_{o p}^{v, 3}=\psi_{o p}^{1}\left(A_{12}, A_{26}, A_{22}, B_{12}, B_{26}, B_{22}\right) \\
& \psi_{o p}^{v, 4}=\psi_{o p}^{2}\left(A_{12}, A_{26}, A_{22}, B_{12}, B_{26}, B_{22}\right), \\
& \psi_{o p}^{\theta^{y}, 1}=\psi_{o p}^{1}\left(B_{11}, B_{16}, B_{22}, D_{12}, D_{16}, D_{22}\right) \\
& \psi_{o p}^{\theta^{y}, 2}=\psi_{o p}^{2}\left(B_{11}, B_{16}, B_{22}, D_{12}, D_{16}, D_{22}\right) \\
& \psi_{o p}^{\theta^{y}, 3}=\psi_{o p}^{1}\left(B_{16}, B_{66}, B_{26}, D_{16}, D_{66}, D_{26}\right)
\end{aligned}
$$




$$
\begin{aligned}
& \psi_{o p}^{\theta^{y}, 4}=\psi_{o p}^{2}\left(B_{16}, B_{66}, B_{26}, D_{16}, D_{66}, D_{26}\right), \\
& \psi_{o p}^{\theta^{x}, 1}=\psi_{o p}^{1}\left(B_{16}, B_{66}, B_{26}, D_{16}, D_{66}, D_{26}\right), \\
& \psi_{o p}^{\theta^{x}, 2}=\psi_{o p}^{2}\left(B_{16}, B_{66}, B_{26}, D_{16}, D_{66}, D_{26}\right), \\
& \psi_{o p}^{\theta^{x}, 3}=\psi_{o p}^{1}\left(B_{12}, B_{26}, B_{22}, D_{12}, D_{26}, D_{22}\right), \\
& \psi_{o p}^{\theta^{x}, 4}=\psi_{o p}^{2}\left(B_{12}, B_{26}, B_{22}, D_{12}, D_{26}, D_{22}\right),
\end{aligned}
$$

for all $o, p \in\{1, \ldots, p+1\}$, where

$$
\begin{aligned}
\psi_{o p}^{1}(a, b, c, d, e, f)=\sum_{m=1}^{p+1} D_{m o} & \left\{\left(a_{o p} \gamma_{11 o p}+b_{o p} \gamma_{21 o p}\right) u_{m p}\right. \\
& +\left(b_{o p} \gamma_{11 o p}+c_{o p} \gamma_{21 o p}\right) v_{m p} \\
& +\left(d_{o p} \gamma_{11 o p}+e_{o p} \gamma_{21 o p}\right) \theta_{m p}^{y} \\
& \left.+\left(e_{o p} \gamma_{11 o p}+f_{o p} \gamma_{21 o p}\right) \theta_{m p}^{x}\right\}, \\
\psi_{o p}^{2}(a, b, c, d, e, f)=\sum_{m=1}^{p+1} D_{m p}\{ & \left(a_{o p} \gamma_{12 o p}+b_{o p} \gamma_{22 o p}\right) u_{o m} \\
& +\left(b_{o p} \gamma_{12 o p}+c_{o p} \gamma_{22 o p}\right) v_{o m} \\
& +\left(d_{o p} \gamma_{12 o p}+e_{o p} \gamma_{22 o p}\right) \theta_{o m}^{y} \\
& \left.+\left(e_{o p} \gamma_{12 o p}+f_{o p} \gamma_{22 o p}\right) \theta_{o m}^{x}\right\},
\end{aligned}
$$

and where the $o p$ subscripts denote evaluation at the associated node.

The transverse-displacement terms, for which all inner products are evaluated with $p \times p$ Gauss-Legendre quadrature, may be written

$$
b_{i j}^{w}=\sum_{\tilde{o}=1}^{p}\left[+D_{i \tilde{o}}\left(\Xi_{j \tilde{o}}^{w, 1}+\Xi_{j \tilde{o}}^{w, 2}\right)+D_{j \tilde{o}}\left(\Xi_{i \tilde{o}}^{w, 3}+\Xi_{i \tilde{o}}^{w, 4}\right)\right],
$$


for all $i, j \in\{1, \ldots, p+1\}$, where the first set of intermediate tensors are

$$
\begin{aligned}
& \Xi_{i \tilde{o}}^{w, 1}=\sum_{\tilde{p}=1}^{p} L_{i \tilde{p}} \gamma_{11 \tilde{o} \tilde{p}}\left[\left(A_{55 \tilde{o} \tilde{p}} \gamma_{11 \tilde{o} \tilde{p}}+A_{45 \tilde{o} \tilde{p}} \gamma_{21 \tilde{o} \tilde{p}}\right) \chi_{\tilde{o} \tilde{p}}^{w, 1}\right. \\
& +\left(A_{55 \tilde{o} \tilde{p}} \gamma_{12 \tilde{o} \tilde{p}}+A_{45 \tilde{o} \tilde{p}} \gamma_{22 \tilde{o} \tilde{p}}\right) \chi_{\tilde{o} \tilde{p}}^{w, 2}+A_{55 \tilde{o} \tilde{p}} \chi_{\tilde{o} \tilde{p}}^{w, 3} \\
& \left.+A_{45 \tilde{o} \tilde{p}} \chi_{\tilde{o} \tilde{p}}^{w, 4}\right] W_{\tilde{o}} W_{\tilde{p}} J_{\tilde{o} \tilde{p}}, \\
& \Xi_{i \tilde{o}}^{w, 2}=\sum_{\tilde{p}=1}^{p} L_{i \tilde{p}} \gamma_{21 \tilde{o} \tilde{p}}\left[\left(A_{45 \tilde{o} \tilde{p}} \gamma_{11 \tilde{o} \tilde{p}}+A_{44 \tilde{o} \tilde{p}} \gamma_{21 \tilde{o} \tilde{p}}\right) \chi_{\tilde{o} \tilde{p}}^{w, 1}\right. \\
& +\left(A_{45 \tilde{o} \tilde{p}} \gamma_{12 \tilde{o} \tilde{p}}+A_{44 \tilde{o} \tilde{p}} \gamma_{22 \tilde{o} \tilde{p}}\right) \chi_{\tilde{o} \tilde{p}}^{w, 2}+A_{45 \tilde{o} \tilde{p}} \chi_{\tilde{o} \tilde{p}}^{w, 3} \\
& \left.+A_{44 \tilde{o} \tilde{p}} \chi_{\tilde{o} \tilde{p}}^{w, 4}\right] W_{\tilde{o}} W_{\tilde{p}} J_{\tilde{o} \tilde{p}}, \\
& \Xi_{i \tilde{o}}^{w, 3}=\sum_{\tilde{p}=1}^{p} L_{i \tilde{p}} \gamma_{12 \tilde{p} \tilde{o}}\left[\left(A_{55 \tilde{p} \tilde{o}} \gamma_{11 \tilde{p} \tilde{o}}+A_{45 \tilde{p} \tilde{o}} \gamma_{21 \tilde{p} \tilde{o}}\right) \chi_{\tilde{p} \tilde{o}}^{w, 1}\right. \\
& +\left(A_{55 \tilde{p} \tilde{o}} \gamma_{12 \tilde{p} \tilde{o}}+A_{45 \tilde{p} \tilde{o}} \gamma_{22 \tilde{p} \tilde{o}}\right) \chi_{\tilde{p} \tilde{o}}^{w, 2}+A_{55 \tilde{p} \tilde{o}} \chi_{\tilde{p} \tilde{o}}^{w, 3} \\
& \left.+A_{45 \tilde{p} \tilde{o}} \chi_{\tilde{p} \tilde{o}}^{w, 4}\right] W_{\tilde{p}} W_{\tilde{o}} J_{\tilde{p} \tilde{o}}, \\
& \Xi_{i \tilde{o}}^{w, 4}=\sum_{\tilde{p}=1}^{p} L_{i \tilde{p}} \gamma_{22 \tilde{p} \tilde{o}}\left[\left(A_{45 \tilde{p} \tilde{o}} \gamma_{11 \tilde{p} \tilde{o}}+A_{44 \tilde{p} \tilde{o}} \gamma_{21 \tilde{p} \tilde{o}}\right) \chi_{\tilde{p} \tilde{o}}^{w, 1}\right. \\
& +\left(A_{45 \tilde{p} \tilde{o}} \gamma_{12 \tilde{p} \tilde{o}}+A_{44 \tilde{p} \tilde{o}} \gamma_{22 \tilde{p} \tilde{o}}\right) \chi_{\tilde{p} \tilde{o}}^{w, 2}+A_{45 \tilde{p} \tilde{o}} \chi_{\tilde{p} \tilde{o}}^{w, 3} \\
& \left.+A_{44 \tilde{p} \tilde{o}} \chi_{\tilde{p} \tilde{o}}^{w, 4}\right] W_{\tilde{p}} W_{\tilde{o}} J_{\tilde{p} \tilde{o}}
\end{aligned}
$$

for all $i \in\{1, \ldots, p+1\}, \tilde{o} \in\{1, \ldots, p\}$, where a tilde over a subscript denotes evaluation of the quantity at the associated Gauss-Legendre quadrature point, and $L_{i \tilde{o}}=\phi_{i}\left(\zeta_{\tilde{o}}\right)$. The intermediate tensors introduced in Eqs. 
(B.26)-(B.29) are given by

$$
\begin{aligned}
\chi_{\tilde{o} \tilde{p}}^{w, 1} & =\sum_{\ell=1}^{p+1} D_{\ell \tilde{o}} \psi_{\ell \tilde{p}}^{w, 1}, & \chi_{\tilde{o} \tilde{p}}^{w, 2} & =\sum_{\ell=1}^{p+1} D_{\ell \tilde{p}} \psi_{\ell \tilde{o}}^{w, 2}, \\
\chi_{\tilde{o} \tilde{p}}^{w, 3} & =\sum_{\ell=1}^{p+1} L_{\ell \tilde{o}} \psi_{\ell \tilde{p}}^{w, 3}, & \chi_{\tilde{o} \tilde{p}}^{w, 4} & =\sum_{\ell=1}^{p+1} L_{\ell \tilde{o}} \psi_{\ell \tilde{p}}^{w, 4},
\end{aligned}
$$

for all $\tilde{o}, \tilde{p} \in\{1, \ldots, p\}$, and where the final set of intermediate tensors are given by

$$
\begin{aligned}
\psi_{\ell \tilde{o}}^{w, 1} & =\sum_{m=1}^{p+1} L_{m \tilde{o}} w_{\ell m}, & \psi_{\ell \tilde{o}}^{w, 2} & =\sum_{m=1}^{p+1} L_{m \tilde{o}} w_{m \ell}, \\
\psi_{\ell \tilde{o}}^{w, 3} & =\sum_{m=1}^{p+1} L_{m \tilde{o}} \theta_{\ell m}^{y}, & \psi_{\ell \tilde{o}}^{w, 4} & =\sum_{m=1}^{p+1} L_{m \tilde{o}} \theta_{\ell m}^{x},
\end{aligned}
$$

for all $\ell \in\{1, \ldots, p+1\}, \tilde{o} \in\{1,2, \ldots, p\}$.

The remaining undefined terms in Eqs. (B.4) and (B.5) are given by

$$
\begin{aligned}
& b_{i j}^{\theta^{y}, S}=\sum_{\tilde{o}=1}^{p} L_{i \tilde{o}} \Xi_{j \tilde{o}}^{\theta^{y}}, \\
& b_{i j}^{\theta^{x}, S}=\sum_{\tilde{o}=1}^{p} L_{i \tilde{o}} \Xi_{j \tilde{o}}^{\theta^{x}},
\end{aligned}
$$

respectively, for all $i, j \in\{1, \ldots, p+1\}$, and the associated intermediate tensors are

$$
\begin{aligned}
& \Xi_{j \tilde{o}}^{\theta^{y}}=\sum_{\tilde{p}=1}^{p} L_{j \tilde{p}} J_{\tilde{o} \tilde{p}} W_{\tilde{o}} W_{\tilde{p}}\left\{+\left(A_{55 \tilde{o} \tilde{p}} \gamma_{11 \tilde{o} \tilde{p}}+A_{45 \tilde{o} \tilde{p}} \gamma_{21 \tilde{o} \tilde{p}}\right) \chi_{\tilde{o} \tilde{p}}^{w, 1}\right. \\
& \left.+\left(A_{55 \tilde{o} \tilde{p}} \gamma_{12 \tilde{o} \tilde{p}}+A_{45 \tilde{o} \tilde{p}} \gamma_{22 \tilde{o} \tilde{p}}\right) \chi_{\tilde{o} \tilde{p}}^{w, 2}+\left(A_{55 \tilde{o} \tilde{p}} \chi_{\tilde{o} \tilde{p}}^{w, 3}+A_{45 \tilde{o} \tilde{p}} \chi_{\tilde{o} \tilde{p}}^{w, 4}\right)\right\} \\
& \Xi_{j \tilde{o}}^{\theta^{x}}=\sum_{\tilde{p}=1}^{p} L_{j \tilde{p}} J_{\tilde{o} \tilde{p}} W_{\tilde{o}} W_{\tilde{p}}\left\{+\left(A_{45 \tilde{o} \tilde{p}} \gamma_{11 \tilde{o} \tilde{p}}+A_{44 \tilde{o} \tilde{p}} \gamma_{21 \tilde{o} \tilde{p}}\right) \chi_{\tilde{o} \tilde{p}}^{w, 1}\right. \\
& \left.+\left(A_{45 \tilde{o} \tilde{p}} \gamma_{12 \tilde{o} \tilde{p}}+A_{44 \tilde{o} \tilde{p}} \gamma_{22 \tilde{o} \tilde{p}}\right) \chi_{\tilde{o} \tilde{p}}^{w, 2}+\left(A_{45 \tilde{o} \tilde{p}} \chi_{\tilde{o} \tilde{p}}^{w, 3}+A_{44 \tilde{o} \tilde{p}} \chi_{\tilde{o} \tilde{p}}^{w, 4}\right)\right\}
\end{aligned}
$$


for all $j \in\{1, \ldots, p+1\}, \tilde{o} \in\{1, \ldots, p\}$.

Through the tensor-product factorization, we have taken the naive matrix-product form, which requires $O\left(p^{6}\right)$ operations per element and negligible storage, and reduced it to $O\left(p^{3}\right)$ operations for each of the terms described above, and $O\left(p^{2}\right)$ storage for each of the intermediate tensors.

[1] ANSYS Mechanical APDL Theory Reference, ANSYS, Inc., 2011.

[2] Abaqus Theory Guide, Dassault Systèmes, 2011.

[3] A. T. Patera, A spectral element method for fluid dynamics: Laminar flow in a channel expansion, Journal of Computational Physics 54 (1984) 468-488.

[4] E. M. Ronquist, A. T. Patera, A Legendre spectral element method for the Stefan problem, International Journal for Numerical Methods in Engineering 24 (1987) 2273-2299.

[5] K. D. Brito, M. A. Sprague, Reissner-Mindlin Legendre spectral finite elements with mixed reduced quadrature, Finite Elements in Analyis and Design 58 (2012) 74-83.

[6] U. Zrahia, P. Bar-Yoseph, Plate spectral elements based upon ReissnerMindlin theory, International Journal for Numerical Methods in Engineering 38 (1995) 1341-1360.

[7] G. Strang, G. J. Fix, An Analysis of the Finite Element Method, Wellesley-Cambridge Press, Wellesley, 1988.

[8] I. Fried, D. S. Malkus, Finite element mass matrix lumping by numerical integration with no convergence rate loss, International Journal of Solids and Structures 11 (1975) 461-466.

[9] P. Kudela, A. Zak, M. Krawczuk, W. Ostachowicz, Modeling of wave propagation in composite plates using the time domain spectral element method, Journal of Sound and Vibration 302 (2007) 728-745.

[10] F. Moleiro, C. M. Mota Soares, C. A. Mota Soares, J. N. Reddy, Mixed least-squares finite element models for static and free vibration analysis of laminated composite plates, Computer Methods in Applied Mechanics and Engineering 198 (2009) 1848-1856. 
[11] F. Moleiro, C. M. Mota Soares, C. A. Mota Soares, J. N. Reddy, Layerwise mixed least-squares finite element models for static and free vibration analysis of multilayered composite plates, Composite Structures 92 (2010) 2328-2338.

[12] Y. Liu, N. Hu, C. Yan, X. Peng, B. Yan, Construction of a Mindlin pseudospectral plate element and evaluating efficiency of the element, Finite Elements in Analysis and Design 45 (2009) 538-546.

[13] N. Hu, Y. Cai, G. Zhu, C. Tsuji, Y. Liu, Alamusi, Y. Cao, Characterization of damage size in metallic plates using Lamb waves, Structural Health Monitoring (2011), 1475921711414230.

[14] T. J. R. Hughes, The Finite Element Method: Linear Static and Dynamic Finite Element Analysis, Prentice-Hall, Inc., Englewood Cliffs, N.J., 1987.

[15] C. Canuto, M. Y. Hussaini, A. Quarteroni, T. A. Zang, Spectral Methods: Fundamentals in Single Domains, Springer-Verlag, Berlin, 2006.

[16] S. A. Orszag, Spectral methods for problems in complex geometries, Journal of Computational Physics 37 (1980) 70-92.

[17] K.-J. Bathe, E. N. Dvorkin, A formulation of general shell elements the use of mixed interpolation of tensorial components, International Journal for Numerical Methods in Engineering 22 (1986) 697-722.

[18] O. O. Ochoa, J. N. Reddy, Finite element analysis of composite laminates, Kluwer Academic Publishers, Dordrecht, 1992.

[19] I. Duff, R. Grimes, J. Lewis, Sparse matrix test problems, ACM Transactions on Mathematical Software 15 (1989) 1-14.

[20] R. D. Cook, D. S. Malkus, M. E. Plesha, R. J. Witt, Concepts and Applications of Finite Element Analysis, 4 ed., Wiley, 2001.

[21] HSL, A collection of fortran codes for large scale scientific computation, 2013. URL: http://www.hsl.rl.ac.uk.

[22] J. N. Reddy, Exact solutions of moderately thick laminated shells, Journal of Engineering Mechanics 5 (1984) 794-809. 
[23] H. Nguyen-Xuan, T. Rabczuk, S. Bordas, J. Debongnie, A smoothed finite element method for plate analysis, Computer Methods in Applied Mechanics and Engineering 197 (2008) 1184-1203.

[24] Q. Wang, M. A. Sprague, Legendre spectral finite element implementation of geometrically exact beam theory, in: Proceedings of the 54th AIAA/ASME/ASCE/AHS/ASC Structures, Structural Dynamics, and Materials Conference, National Harbor, MD, 2013.

[25] Q. Wang, M. A. Sprague, J. Jonkman, N. Johnson, Nonlinear Legendre spectral finite elements for wind turbine blade dynamics, in: Proceedings of the 32nd ASME Wind Energy Symposium, AIAA Science and Technology Forum and Exposition, Boston, MA, 2014.

[26] M. O. Deville, P. F. Fischer, E. H. Mund, High-Order Methods for Incompressible Fluid Flow, Cambridge University Press, 2002. 